\title{
High-dose steroids for the treatment of severe COVID-19
}

\author{
Matteo Piccica ${ }^{1} \cdot$ Filippo Lagi $^{1,2} \cdot$ Michele Trotta $^{2} \cdot$ Michele Spinicci $^{1,2} \oplus \cdot$ Lorenzo Zammarchi $^{1,2}$. \\ Alessandro Bartoloni ${ }^{1,2} \cdot$ For the COCORA Working Group
}

Received: 29 December 2020 / Accepted: 8 March 2021 / Published online: 15 April 2021

(c) Società Italiana di Medicina Interna (SIMI) 2021

\section{Dear Editor,}

Twelve months after the beginning of the Coronavirus disease 2019 (COVID-19) pandemic, there is no established therapy for patients with severe acute respiratory syndrome Coronavirus 2 (SARS-CoV-2) infection [1, 2]. Glucocorticosteroids (called steroids thereafter) are the only drug that demonstrated to reduce mortality and the need for invasive mechanical ventilation in hospitalized patients with COVID19 [3, 4]. Accordingly, several national and international guidelines included systemic steroids for the treatment of severe COVID-19 patients [5]. For instance, World Health Organization (WHO) and UK National Institute for Health and Care Excellence strongly recommend low-dose steroids (namely dexamethasone $6 \mathrm{mg} /$ die or equivalent dose) for 7-10 days in adults with severe or critical disease [2, 6].

The optimal dose and duration of steroids treatment are mainly based on a single clinical trial (RECOVERY trial) [7]. However, a conclusive comparison of the efficacy between high- and low-dose steroids in COVID-19 patients is not available to date. In other settings, steroids therapy for moderate-to-severe acute respiratory distress syndrome (ARDS) has been recommended at a higher dose [8]. Therefore, the hypothesis that a higher dose could be beneficial, at least in critically ill COVID-19 patients, deserves to be explored.

In this case series, we reported clinical characteristics, outcome, and side effects of a small population of patients

Members of "For the COCORA Working Group" are listed in Acknowledgement section.

Michele Spinicci

michele.spinicci@unifi.it

1 Department of Experimental and Clinical Medicine, SOD Malattie Infettive eTropicali, University of Florence, Largo Brambilla 3, 50134 Florence, Italy

2 Infectious and Tropical Diseases Unit, Azienda Ospedaliero-Universitaria Careggi, Florence, Italy with critical COVID-19, treated with a high-dose steroids (methylprednisolone equivalent $\geq 2 \mathrm{mg} / \mathrm{kg} /$ day) at Careggi University Hospital, Florence, Italy, during the period February 25-April 25, 2020, in three internal medicine and one infectious diseases unit.

Inclusion criteria were: (1) diagnosis of COVID-19 confirmed by at least one positive result of real-time polymerase chain reaction (RT-PCR) in a diagnostic specimen (nasopharyngeal swab, sputum, broncho-alveolar lavage); (2) treatment with at least one dose of intravenous high-dose steroids, defined as methylprednisolone equivalent $\geq 2 \mathrm{mg}$ / $\mathrm{kg}$ /day; (3) oxygenation impairment consistent with partial pressure to fractional inspired oxygen ratio $\left(\mathrm{PaO}_{2} / \mathrm{FiO}_{2}\right)$ less than $200 \mathrm{mmHg}$, before the administration of high-dose steroids. An improved patient was defined as a discharged patient with no need for supplementary oxygen, irrespectively from the monitoring RT-PCR results.

Viral clearance was defined as two negative result at RTPCR for SARS-CoV-2 in a nasopharyngeal swab. Descriptive analysis was employed to illustrate population characteristics. Data collection was approved by the local Ethics Committee (17104_oss). The study was performed following the ethical principles of the Declaration of Helsinki and with the International Conference on Harmonization Good Clinical Practice guidelines.

Overall, we evaluated 397 patients. Of them, 95 (23.9\%) were treated with steroids, but only 14 (3.5\%) patients received a high-dose steroids (as for the inclusions criteria); 1 patient was excluded from the analysis because of the $\mathrm{PaO}_{2} / \mathrm{FiO}_{2}$ above $200 \mathrm{mmHg}$.

Thus, we included 13 patients. The median age was 76 years (IQR 62-83), 7 (53.8\%) were male. The median Charlson comorbidity index (CCI) was 4 (IQR 3-4), and 4 of them had a do-not-resuscitate (DNR) status, according to the medical condition. The median $\mathrm{PaO}_{2} / \mathrm{FiO}_{2}$ at the hospitalization and before starting high-dose steroids was $229 \mathrm{mmHg}$ (IQR 134-293) and $98 \mathrm{mmHg}$ (IQR 61-138), respectively (Table 1). Eleven patients had bilateral pulmonary infiltrates, demonstrated by chest radiography and/or high-resolution 
Table 1 Demographic and clinical characteristics of COVID-19 population treated with high-dose steroid

\begin{tabular}{|c|c|}
\hline Characteristics & Patients $(n=13)$ \\
\hline Age (median, IQR), years & $76(62-83)$ \\
\hline \multicolumn{2}{|l|}{ Male gender } \\
\hline Male & $7(53.8 \%)$ \\
\hline \multicolumn{2}{|l|}{ Comorbidities } \\
\hline Hypertension & $7(53.8 \%)$ \\
\hline Diabetes & $1(7.6 \%)$ \\
\hline Chronic obstructive pulmonary disease & $2(15.4 \%)$ \\
\hline Malignancies & $2(15.4 \%)$ \\
\hline Coronary artery diseases & $1(7.6 \%)$ \\
\hline Charlson comorbidities index (median, IQR) & $4(3-4)$ \\
\hline \multicolumn{2}{|l|}{ Smoking history } \\
\hline Active smokers & $0(0 \%)$ \\
\hline Former smokers & $6(46.1 \%)$ \\
\hline Non-smokers & $5(38.5 \%)$ \\
\hline Not know & $2(15.4 \%)$ \\
\hline \multicolumn{2}{|l|}{ Symptoms } \\
\hline Cough & $9(69.2 \%)$ \\
\hline Fever & $11(84.6 \%)$ \\
\hline Diarrhea & $3(23.1 \%)$ \\
\hline Dyspnoea & $5(38.5 \%)$ \\
\hline \multicolumn{2}{|l|}{ Radiological features } \\
\hline Chest X-ray bilateral infiltrates & $11(84.6 \%)$ \\
\hline HRCT bilateral ground glass opacities & $4(30.7)$ \\
\hline $\begin{array}{l}\text { Time from onset of symptoms to high-dose steroid } \\
\text { administration (median, IQR), days }\end{array}$ & $14(10-16)$ \\
\hline Hydroxychloroquine & $12(92.3 \%)$ \\
\hline Protease inhibitors & $11(84.6 \%)$ \\
\hline Tocilizumab & $5(38.5 \%)$ \\
\hline Ruxolitinib & $1(7.6 \%)$ \\
\hline $\mathrm{PaO}_{2} / \mathrm{FiO}_{2}$ at the admission (median, IQR) & $229(134-293)$ \\
\hline $\mathrm{PaO}_{2} / \mathrm{FiO}_{2}$ before high-dose steroid (median, IQR) & $98(61-138)$ \\
\hline \multicolumn{2}{|l|}{ Oxygen supportive therapy } \\
\hline Ventury-mask only & $5(38.5 \%)$ \\
\hline High flow nasal cannula (HFNC) only & $2(15.4 \%)$ \\
\hline Non invasive ventilation (NIV) only & $2(15.4 \%)$ \\
\hline HFNC and NIV & $4(30.8 \%)$ \\
\hline \multicolumn{2}{|l|}{ Outcome } \\
\hline Improved & $9(69.2 \%)$ \\
\hline Deceased & 4/13 (30.8\%) \\
\hline
\end{tabular}

$I Q R$ interquartile ranges, HRCT high-resolution chest tomography, $\mathrm{PaO}_{2} / \mathrm{FiO}_{2}$ partial pressure to fractional inspired oxygen ratio

chest tomography. In comparison with patients receiving low-dose steroids, those treated with high doses had lower $\mathrm{PaO}_{2} / \mathrm{FiO}_{2}$ at the admission (229 [IQR 134-293] vs. 296 [255-333], $p=0.004)$, and received steroids in a later stage (14 vs. 10 days from symptoms onset, $p=0.022$ ), when ARDS was more frequently present (100\% vs. $67 \%$, $p=0.016$ ). Among patients receiving high-dose steroids, nine improved (69.2\%) and four deceased (30.8\%); patients treated with low-dose steroids had a higher mortality (31/81, $38.3 \%$ ), without reaching a statistically significant difference $(\mathrm{p}=0.625)$ (data not shown).

Among the nine improved patients, seven achieved the viral clearance after a median of 16 days (IQR 10-33), while in two cases date of viral clearance was missing. The median time between the onset of symptoms and high-dose steroids administration was 14 days (IQR 10-16).

Steroids were administered for a median of 4 days (IQR $3-5)$; the median dosage of methylprednisolone used was $250 \mathrm{mg}$ (IQR 130-271), and the median dosage adjusted for body weight was $2.6 \mathrm{mg} / \mathrm{kg} / \mathrm{die}$ (IQR 2.13-3.71). At the time of the first steroids infusion, two patients (15.3\%) had fever. Of note, five patients (38.5\%) also received tocilizumab and one $(7.7 \%)$ ruxolitinib. Detailed information on clinical features, therapies and outcome of each patient treated with high-dose steroids are reported in Table 2.

About supportive oxygen therapy, five (38.5\%) patients received Venturi-mask, while eight $(61.5 \%)$ required highflow-nasal cannulas (HFNC) and/or additional non-invasive ventilation (NIV). No one of the included patients was intubated nor admitted to the intensive care unit. Among the improved patients, the median time from the first steroids dose to exceed $\mathrm{PaO}_{2} / \mathrm{FiO}_{2}>200 \mathrm{mmHg}$ was 6 days (IQR 6-10 days). Trends of CRP, following high-dose steroids administration, are shown in Fig. 1.

No grade 3-5 adverse events were registered after steroids administration: only one patient developed an uncomplicated urinary tract infection (UTI) 4 days after receiving the first steroids dose; three patients developed alteration of blood glucose ( $>250 \mathrm{mg} / \mathrm{dL}$ ), while four developed minor alteration (126-249 mg/dL). In all cases, the glucose levels returned into the normal range within 2 weeks from the steroid discontinuation, without the use of antidiabetic drugs. No clinical suspicion of femoral head necrosis was observed.

Currently, no evidence suggested that higher doses of steroids are associated with more significant benefits than a lower dose. Our study aims to explore whether the use of high-dose steroids could be a possible option to consider in the weaponry against COVID-19, especially in critically ill patients. The rationale of their use is to tackle "cytokine storm"-related manifestations, and it partially derives from data obtained during the SARS-CoV epidemic of 2003, when systemic steroids were administered in infected patients who developed the severe respiratory disease [9, 10]. Another relevant point is that early high-dose steroids 


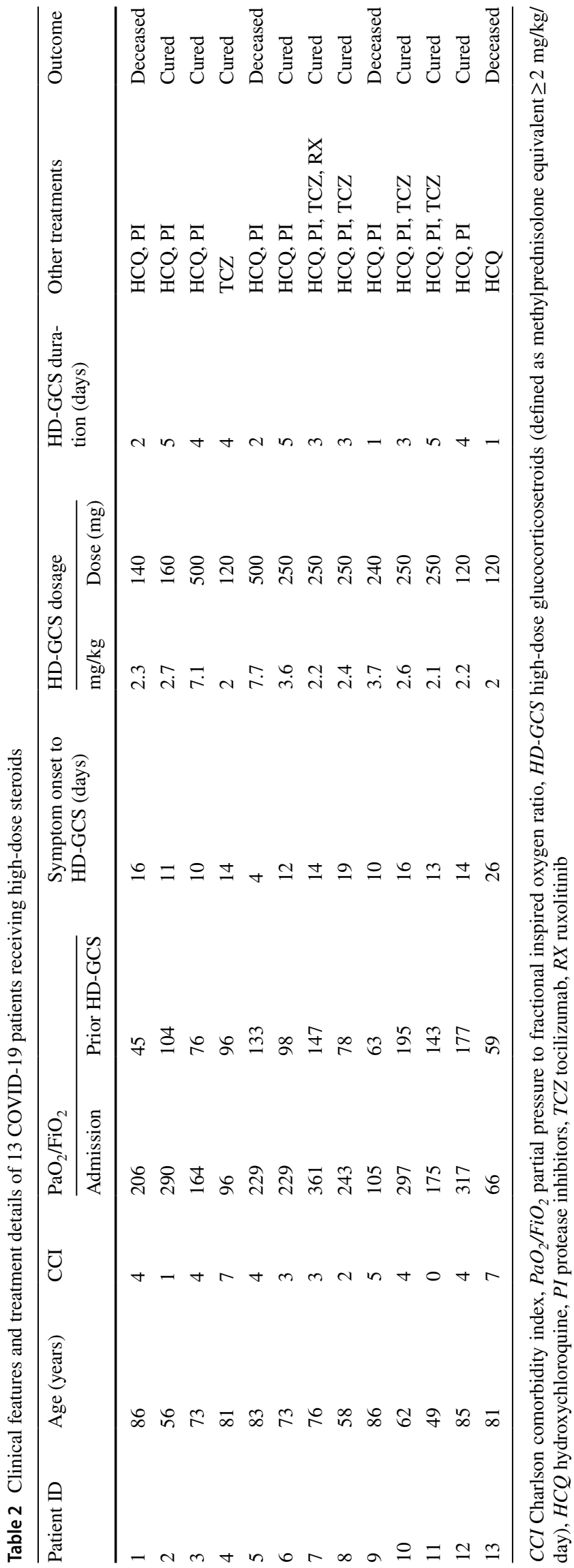

could preserve pulmonary fibrosis and long-term pulmonary consequences in the surviving patients [11].

The main limitation of our study is the small number of included subjects and its retrospective design. For this reason, an exhaustive evaluation of the efficacy of high-dose steroids in this setting is beyond the scope of the paper.

The steroids dosage utilized in our case series was much higher (a median 2.6 (IQR 2.13-3.71) mg/kg/die of 6-methylprednisolone) than that currently recommended in the most relevant international guidelines, and that reported in the most of the published studies [7, 12]. SARS-CoV-2-related ARDS is characterized by the poor outcome, with mortality above $50 \%$ in the short term [13]. According to our findings, among 13 patients with critical COVID-19, 9 (69.2\%) improved, and 4 (30.8\%) died; no one was admitted in the intensive care unit. Moreover, all patients with an unfavorable outcome were over 80 years old, had DNR status, and started the steroids treatment when $\mathrm{PaO}_{2} / \mathrm{FiO}_{2}$ was $<150 \mathrm{mmHg}$. In particular, two of them received steroids in a very late stage of the disease (namely, patient no. 13, 21 days after the first record of $\mathrm{PaO}_{2} / \mathrm{FiO}_{2}<200 \mathrm{mmHg}$ and patient no. 5, in a condition of severe hypercapnia, respiratory acidosis, and Glasgow Come Scale impairment).

Although the use of high-dose steroids is known to be burdened by serious side effects, in our experience, use of high-dose steroids therapy resulted safe and well tolerated. Altered blood glucose level is the main side effect observed. In all cases, this condition resolved spontaneously, without the need for antidiabetic drugs.

Concerning the timing of viral clearance, no substantial difference was seen between our population (16 days) compared to the available data in the literature: for instance, in a Chinese cohort of patients with COVID-19, the median time of viral shedding was 20 days (IQR 17-24), without receiving steroids [14].

In conclusion, our preliminary results support the usefulness of a randomized clinical trial, investigating the impact of different dosage of steroids on the outcome of critical COVID-19 patients. Furthermore, future studies are needed to identify which patient could benefit from high-dose steroids, to investigate the additional effect of other immunomodulant drugs, such as tocilizumab or JAK inhibitors, and to disclose whether steroids treatment could prevent or reduce the risk for pulmonary fibrosis or any residual lung impairment after SARS-CoV-2 infection. 


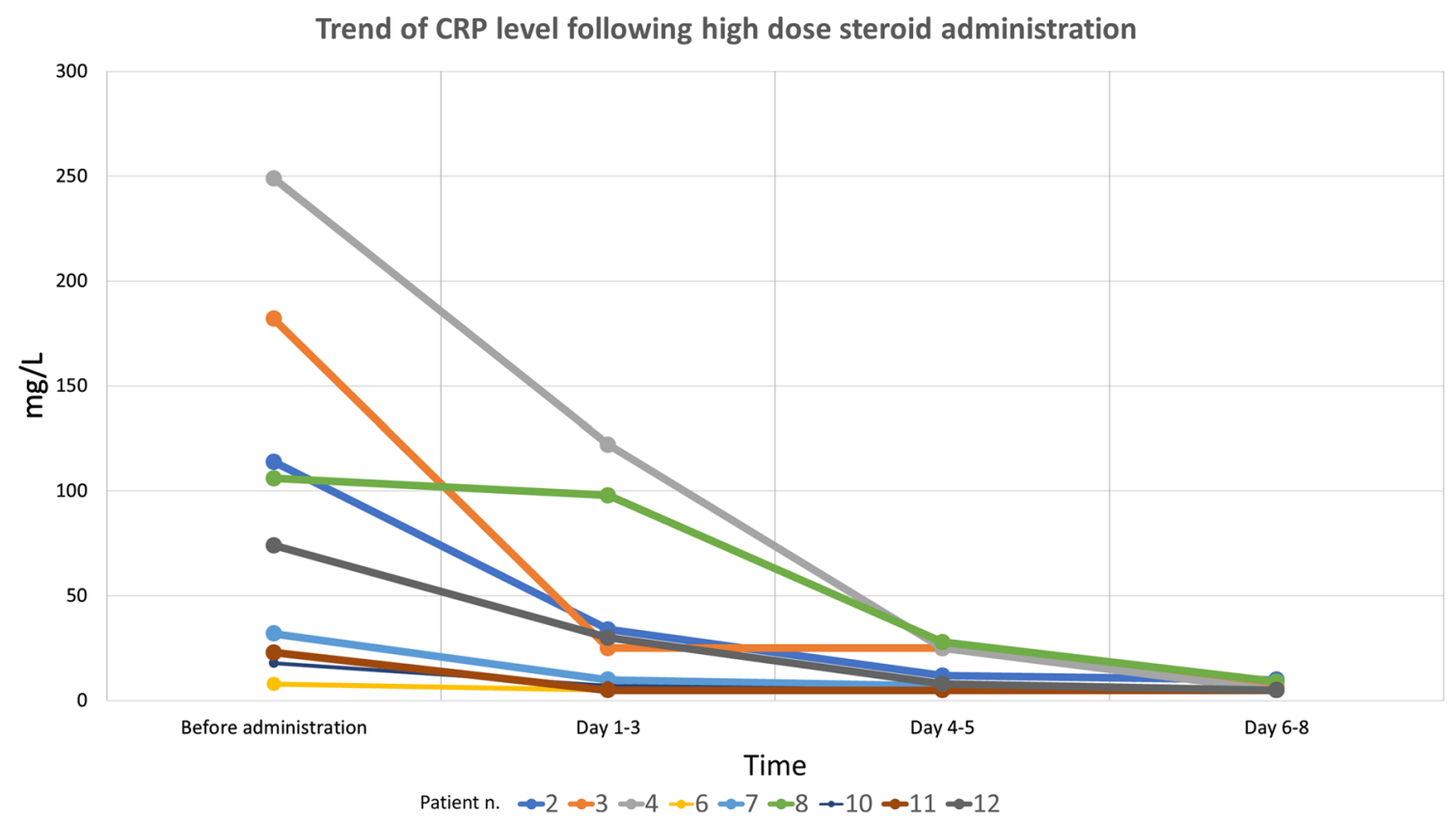

Fig. 1 Temporal trends of C-reactive protein for ten patient treated with high-dose steroid

\section{Acknowledgements For the COCORA Working Group}

Filippo Lagi, Matteo Piccica, Lucia Graziani, Iacopo Vellere, Annarita Botta, Marta Tilli, Letizia Ottino, Beatrice Borchi, Marco Pozzi, Filippo Bartalesi, Jessica Mencarini, Michele Spinicci, Lorenzo Zammarchi, Filippo Pieralli, Giovanni Zagli, Carlo Nozzoli, Stefano Romagnoli, Alessandro Bartoloni, Irene Campolmi, Nicoletta Di Lauria, Giovanni Millotti, Gregorio Basile, Massimo Meli, Pier Giorgio Rogasi, Dario Bartolozzi, Paola Corsi, Marcello Mazzetti, Alberto Farese, Silvia Bresci, Annalisa Cavallo, Michele Trotta, Giampaolo Corti, Alessandro Morettini, Loredana Poggesi, Angelo Raffaele De Gaudio, Adriano Peris, Paolo Fontanari, Paola Parronchi, Fabio Almerigogna, Francesco Annunziato, Francesco Liotta, Lorenzo Cosmi, Alessandra Vultaggio, Andrea Matucci, Manuela Angileri, Alessandra Ipponi, Michele Cecchi, Silvia Benemei, Alessandro Maria Vannucchi, Marco Matucci-Cerinic, Lucia Turco.

Funding This work was supported by funds of Ministry of Education, University and Research (Italy) Excellence Departments 2018-2022 (Project for the Department of Experimental and Clinical Medicine).

Availability of data and materials Most data are available in the text and in the supplementary material. Further data will be provided on request.

\section{Declarations}

Conflict of interest The authors declared no conflicts of interest.

Ethics approval Data collection was approved by the local Ethics Committee (17104_oss). The study was performed in accordance with the ethical principles of the Declaration of Helsinki and with the International Conference on Harmonization Good Clinical Practice guidelines.

Consent to participate The study was performed as a clinical audit using routine collected clinical data in an anonymised format, and as such is exempt from the need to take specific written informed consent.

\section{References}

1. Polosa R, Spinicci M, Prisco D (2020) "COVID-19: diagnosis, managment and prognosis: a new topical collection of Internal and Emergency Medicine. Intern Emerg Med 15(5):747-750

2. Lagi F, Piccica M, Graziani L et al (2020) Early experience of an infectious and tropical diseases unit during the coronavirus disease (COVID-19) pandemic, Florence, Italy, February to March 2020. Euro Surveill 25(17):2000556

3. World Health Organization. Corticosteroids for COVID-19 (2020) WHO Living Guidance. https://www.who.int/publications/i/item/ WHO-2019-nCoV-Corticosteroids-2020.1. Accessed 28th Dec 2020

4. Sterne JAC, Murthy S, Diaz JV et al (2020) Association between administration of systemic corticosteroids and mortality among critically Ill patients with COVID-19 a meta-analysis. JAMA 324(13):1330-1341

5. British Medical Journal best practice (2020) Coronavirus disease 2019 (COVID-19). Last updated: Dec 21, 2020. https://bestpracti ce.bmj.com/topics/en-gb/3000201/management-recommenda tions. Accessed on 28th Dec 2020

6. National Institute for Health and Care Excellence (2020) COVID19 prescribing briefing: corticosteroids. Last updated: October 2020. https://www.nice.org.uk/guidance/ng159/resources/ covid19-prescribing-briefing-corticosteroids-pdf-8839913581. Accessed on 28th Dec 2020

7. Horby P, Shen Lim W, Emberson J et al (2020) Dexamethasone in Hospitalized Patients with Covid-19-Preliminary Report. N Engl J Med:1-11

8. Steinberg KP, Hudson LD, Goodman RB et al (2006) Efficacy and safety of corticosteroids for persistent acute respiratory distress syndrome. N Engl J Med:1671-1684

9. Cain DW, Cidlowski JA (2017) Immune regulation by glucocorticoids. Nat Publ Gr 17(4):233-247

10. Stockman LJ, Bellamy R, Garner P (2006) SARS : systematic review of treatment effects. PLoS Med 3(9) 
11. Spagnolo P, Balestro E, Aliberti S et al (2020) Pulmonary fibrosis secondary to COVID-19: a call to arms ? Lancet Respir 2019(20):2019-2020

12. Corral L, Bahamonde A, Arnaiz F et al (2020) GLUCOCOVID: a controlled trial of methylprednisolone in adults hospitalized with COVID-19 pneumonia. medRxyv. https://www.medrxiv. org/content/https://doi.org/10.1101/2020.06.17.20133579v1 (preprint)

13. Wu C, Chen X, Cai Y et al (2020) Risk factors associated with acute respiratory distress syndrome and death in patients with Coronavirus Disease 2019 Pneumonia in Wuhan, China. JAMA Intern Med:e200994
14. Zhou F, Yu T, Du R et al (2020) Clinical course and risk factors for mortality of adult inpatients with COVID-19 in Wuhan, China : a retrospective cohort study. Lancet 6736(20):1-9

Publisher's Note Springer Nature remains neutral with regard to jurisdictional claims in published maps and institutional affiliations. 